\title{
Optimal Investment with Multiple Risky Assets for an Insurer in an Incomplete Market
}

\author{
Hui Zhao, ${ }^{1}$ Ximin Rong, ${ }^{1,2}$ and Jiling $\mathrm{Cao}^{3}$ \\ ${ }^{1}$ School of Science, Tianjin University, Tianjin 300072, China \\ ${ }^{2}$ Center for Applied Mathematics, Tianjin University, Tianjin 300072, China \\ ${ }^{3}$ School of Computing and Mathematical Sciences, Auckland University of Technology, Private Bag 92006, Auckland 1142, New Zealand
}

Correspondence should be addressed to Ximin Rong; rongximin@tju.edu.cn

Received 30 November 2012; Accepted 6 February 2013

Academic Editor: Xiaochen Sun

Copyright (C) 2013 Hui Zhao et al. This is an open access article distributed under the Creative Commons Attribution License, which permits unrestricted use, distribution, and reproduction in any medium, provided the original work is properly cited.

\begin{abstract}
This paper studies the optimal investment problem for an insurer in an incomplete market. The insurer's risk process is modeled by a Lévy process and the insurer is supposed to have the option of investing in multiple risky assets whose price processes are described by the standard Black-Scholes model. The insurer aims to maximize the expected utility of terminal wealth. After the market is completed, we obtain the optimal strategies for quadratic utility and constant absolute risk aversion (CARA) utility explicitly via the martingale approach. Finally, computational results are presented for given raw market data.
\end{abstract}

\section{Introduction}

Recently, the problem of optimal investment for an insurer has attracted a lot of attention, due to the fact that the insurer is allowed to invest in financial markets in practice. In the meantime, this is also a very interesting portfolio selection problem in the finance theory. Some important early work is done by Browne [1], where the risk process is approximated by a Brownian motion with drift and the stock price is modeled by a geometric Brownian motion. Under these assumptions, when the interest rate of a risk-free bond is zero, Browne [1] shows that minimizing the ruin probability and maximizing the expected constant absolute risk aversion (CARA) utility of the terminal wealth produce the same type of strategy. In Hipp and Plum [2], the classical Cramér-Lundberg model is adopted for the risk reserve and the insurer can invest in a risky asset to minimize the ruin probability. However, the interest rate of the bond in their model is implicitly assumed to be zero. Liu and Yang [3] extend the model of Hipp and Plum [2] to incorporate a nonzero interest rate. But in this case, a closed-form solution cannot be obtained. Azcue and Muler [4] consider the borrowing constraints for an optimal investment problem of an insurer, and some numerical solutions to minimize the ruin probability are obtained. In the literature, the optimal investment problem for an insurer is usually studied via the stochastic control theory; see [5] for details. By this approach, Yang and Zhang [6] use a jump-diffusion process to model the risk process and obtain the optimal investment strategy for CARA utility maximization. The martingale approach, which has been widely used in mathematical finance, is applied to investigate the optimal investment problem for an insurer by Wang et al. [7]. In their paper, the risk process is modeled by a Lévy process, the security market containing a bond and a stock is described by the standard Black-Scholes model, and the optimal strategies are worked out explicitly for the meanvariance criteria and the CARA utility. Zhou [8] studies a model similar to that in [7], where the prices of assets are described by the Lévy processes, and the optimal strategy is obtained explicitly for the CARA utility.

In most of these papers, the optimal investment for an insurer is considered in a complete market. But the real market is always incomplete; that is, the number of risky assets (stocks) is strictly less than the dimension of the underlying Brownian motion. Hence, it is necessary to find an optimal strategy in an incomplete market. However, the traditional martingale method cannot be used directly in an incomplete market. To overcome the problem of incompleteness, many 
researchers have developed different ways to handle the problem. For instance, Karatzas et al. [9] complete the market by introducing additional fictitious stocks and then making them untradable. Zhang [10] provides an easier method by directly making the dimension of the underlying Brownian motion equal to the number of available stocks.

The optimal investment problem for an insurer in an incomplete market is studied in the present paper. For this case, the traditional martingale method is problematic. Thus, we first complete the market via the approach proposed by Zhang [10] so that the martingale method can be used. In addition, only one stock is considered in $[7,8]$. But an insurer will invest in multiple stocks to decrease risk. Thus in order to make our model more practical, we consider a financial market of one bond and $m$ stocks. Moreover, the insurer's objective in this paper is to maximize the utility of the discounted value of his/her terminal wealth, which is different from that in most works. When the risk process is modeled by a Lévy process and the security market is described by the standard Black-Scholes model, the optimal strategies are obtained explicitly for the quadratic and CARA utilities.

An optimal strategy of CARA utility maximizing in an incomplete market is also obtained in Wang [11]. The result is general but not unique and cannot be expressed concretely in [11]. Whereas our corresponding optimal strategy is explicit and concrete.

This paper is structured as follows. In Section 2 the problem is formulated. The incomplete market is transformed into a complete one in Section 3. The optimal portfolios for quadratic and CARA utility functions are worked out explicitly in Section 4. Section 5 presents some remarks and computational results for optimal strategies in a complete market and an incomplete market. Section 6 contains some conclusions.

\section{Formulation of the Problem}

The insurer can invest in a financial market consisting of one riskless bond with price $S_{t}^{(0)}$ given by

$$
\mathrm{d} S_{t}^{(0)}=r S_{t}^{(0)} \mathrm{d} t, \quad S_{0}^{(0)}=1,
$$

and $m$ stocks with prices $S_{t}^{(i)}$ satisfying

$$
\mathrm{d} S_{t}^{(i)}=S_{t}^{(i)}\left[b_{i} \mathrm{~d} t+\sum_{j=1}^{d} \sigma_{i j} \mathrm{~d} W_{t}^{(j)}\right], \quad i=1, \ldots, m,
$$

where $r$ is the interest rate and $W_{t}:=\left(W_{t}^{(1)}, \ldots, W_{t}^{(d)}\right)^{T}$ is a $d$-dimensional Brownian motion defined on a filtered complete probability space $\left(\Omega, \mathscr{F},\left(\mathscr{F}_{t}\right), \mathbb{P}\right)$ with the component Brownian motions $W_{t}^{(j)}, j=1, \ldots, d$ being independent. $b:=\left(b_{1}, \ldots, b_{m}\right)^{T}$ denotes the appreciation rate vector, $\sigma:=$ $\left(\sigma_{i j}\right)_{m \times d}$ is the volatility matrix, and define $T>0$ as a fixed and finite time horizon with $0 \leq t \leq T$. In addition, the matrix $\sigma$ is assumed to have the full row rank. As in [10], under these assumptions, we restrict ourselves to the situation where $m \leq d$ that is, the number of stocks in the market is smaller than or equal to the dimension of the underlying Brownian motion. This financial market is complete when $m=d$, while incompleteness arises if $m<d$. In this paper, we consider the insurer's investment problem in an incomplete market.

Suppose the initial reserve is $x_{0}$ and the premium rate is a constant $\alpha$; the risk process $\left(R_{t}\right)$ of the insurer is modeled by

$$
R_{t}=x_{0}+\alpha t-\sum_{i=1}^{N(t)} Y_{i}
$$

where $\sum_{i=1}^{N(t)} Y_{i}$ is a compound Poisson process defined on $(\Omega, \mathscr{F}, \mathbb{P}), N(t)$ is a homogeneous Poisson process with intensity $\lambda$ and represents the number of claims occurring in the time interval $[0, t], Y_{i}$ is the size of the $i$ th claim. Thus the compound Poisson process $\sum_{i=1}^{N(t)} Y_{i}$ represents the cumulative amount of claims in the time interval $[0, t]$. The claims' sizes $Y=\left\{Y_{i}, i \geq 1\right\}$ are assumed to be an i.i.d. sequence with a common cumulative distribution function $F$ satisfying $F(0)=0$ and $\int_{0}^{\infty} x^{2} \mathrm{~d} F(x)<\infty$. Furthermore, it is usually assumed that $N,\left\{Y_{i}, i \geq 1\right\}$, and the $d$-dimensional Brownian motion $W$ are mutually independent. If $L_{t}$ denotes the compensated compound Poisson process $\sum_{i=1}^{N(t)} Y_{i}-\lambda m_{F} t$, where $m_{F}$ is the mean of $F$, then the risk process (3) can be rewritten as

$$
R_{t}=x_{0}+\left(\alpha-\lambda m_{F}\right) t-L_{t}
$$

As in [7], we generalize the above risk process model to a stochastic cash flow, which is still denoted by $\left(R_{t}\right)$ and satisfies

$$
R_{t}=x_{0}+c t-L_{t},
$$

where $L$ is a 1-dimensional compensated pure jump Lévy process defined on $\left(\Omega, \mathscr{F}_{,}\left(\mathscr{F}_{t}\right), \mathbb{P}\right), c$ is a constant, $\left(\mathscr{F}_{t}\right)$ is the usual augmentation of the natural filtration of $(W, L)$ with $\mathscr{F}=\mathscr{F}_{T}$, and $W$ and $L$ are mutually independent. Let $\mu$ denote the jump measure of $L$. Its dual predictable projection $v$ has the form $v(\mathrm{~d} t, \mathrm{~d} x)=\mathrm{d} t \times m(\mathrm{~d} x)$ with $m(\{0\})=0$ and $\int_{\mathbb{R}}\left(x^{2} \wedge 1\right) m(\mathrm{~d} x)<\infty$. To avoid some tedious technical arguments, we assume $\int_{\mathbb{R}} x^{2} m(\mathrm{~d} x)<\infty$ throughout this paper. Under this assumption, it is well known that $L$ is square integrable and has the following Lévy decomposition property:

$$
L_{t}=\int_{0}^{t} \int_{\mathbb{R}} x(\mu(\mathrm{d} s, \mathrm{~d} x)-v(\mathrm{~d} s, \mathrm{~d} x)) .
$$

For details, refer to Cont and Tankov [12]. Further, note that for the risk process model (3), we have $m(\mathrm{~d} x)=\lambda F(\mathrm{~d} x)$.

The insurer is allowed to invest in those $m$ stocks as well as in the bond. A trading strategy can be expressed in terms of the dollar amount invested in each asset. Let $\pi_{t}^{(i)}, i=1, \ldots, m$, be the dollar amount of the $i$ th stock in the investment portfolio at time $t$, and let $\pi_{t}:=\left(\pi_{t}^{(1)}, \ldots, \pi_{t}^{(m)}\right)^{T}$ and $\pi:=\left(\pi_{t}\right)$. To be mathematically rigorous, for each $1 \leq$ $i \leq m, \pi_{t}^{(i)}$ is an $\left(\mathscr{F}_{t}\right)$-predictable process. 
Definition 1. A trading strategy $\pi$ is called admissible if $\mathbb{E}\left[\int_{0}^{T} \pi_{t}^{T} \pi_{t} \mathrm{~d} t\right]<\infty$

The set of all admissible trading strategies is denoted by $\Pi$. For each $\pi \in \Pi$ and an initial capital $x_{0}$, the wealth process $X^{x_{0}, \pi}$ of the insurer satisfies the following dynamics:

$$
\begin{gathered}
\mathrm{d} X_{t}^{x_{0}, \pi}=\pi_{t}^{T}\left[b \mathrm{~d} t+\sigma \mathrm{d} W_{t}\right]+\left(X_{t}^{x_{0}, \pi}-\pi_{t}^{T} \mathbf{1}_{m}\right) r \mathrm{~d} t \\
+c \mathrm{~d} t-\mathrm{d} L_{t} \\
\quad X_{0}^{x_{0}, \pi}=x_{0},
\end{gathered}
$$

where $\mathbf{1}_{m}:=(1, \ldots, 1)^{T}$ is an $m \times 1$ vector. Wang et al. [7] consider an optimal investment problem similar to (7) in the one stock case and solve it elegantly by the martingale method in a complete market. However, in an incomplete market, the martingale method will be problematic. Thus, we will use the method proposed by Zhang [10] to transform our incomplete market into a complete one.

\section{Transformation from an Incomplete Market to a Complete One}

In this section, we "complete" the incomplete market described in (1) and (2) in order to use the martingale method in the sequel.

Step 1. Reducing the dimension of the Brownian motion.

For each $i=1, \ldots, m$, let $\sigma_{i}:=\left(\sigma_{i 1}, \sigma_{i 2}, \ldots, \sigma_{i d}\right)$. Then the volatility matrix in the incomplete market can be written as $\sigma=\left(\sigma_{1}, \sigma_{2}, \ldots, \sigma_{m}\right)^{T}$. Define

$$
B_{t}^{(i)}:=\sum_{j=1}^{d} \frac{\sigma_{i j}}{\left\|\sigma_{i}\right\|} W_{t}^{(j)}, \quad i=1, \ldots, m,
$$

where $\|\cdot\|$ denotes the Euclidean norm. Expressing the stock prices in terms of $B_{t}^{(i)}$, it gives

$$
\mathrm{d} S_{t}^{(i)}=S_{t}^{(i)}\left(b_{i} \mathrm{~d} t+\left\|\sigma_{i}\right\| \mathrm{d} B_{t}^{(i)}\right), \quad i=1, \ldots, m,
$$

where $\left(B_{t}^{(1)}, \ldots, B_{t}^{(m)}\right)^{T}$ is an $m$-dimensional Brownian motion. But the component Brownian motions are no longer independent. Specifically, one can consult Exercise 4.15 in [13] for the following result:

$$
\mathrm{d} B_{t}^{(i)} \mathrm{d} B_{t}^{(k)}=\rho_{i k} \mathrm{~d} t, \quad \forall i \neq k,
$$

where

$$
\rho_{i k}=\frac{1}{\left\|\sigma_{i}\right\|\left\|\sigma_{k}\right\|} \sum_{j=1}^{d} \sigma_{i j} \sigma_{k j}=\frac{\left\langle\sigma_{i}, \sigma_{k}\right\rangle}{\left\|\sigma_{i}\right\|\left\|\sigma_{k}\right\|},
$$

and $\left\langle\sigma_{i}, \sigma_{k}\right\rangle$ represents the inner product of $\sigma_{i}$ and $\sigma_{k}$. It follows from the Cauchy-Schwarz inequality that $\left|\rho_{i k}\right| \leq 1$. Under the assumption that the volatility matrix has the full row rank, we have

$$
\left|\rho_{i k}\right|<1 \quad \text { for } i \neq k
$$

Step 2. Creating independent component Brownian motions from the correlated ones.

Let

$$
\Psi:=\left[\begin{array}{cccc}
\rho_{11} & \rho_{12} & \cdots & \rho_{1 m} \\
\rho_{21} & \rho_{22} & \cdots & \rho_{2 m} \\
\vdots & \vdots & \ddots & \vdots \\
\rho_{m 1} & \rho_{m 2} & \cdots & \rho_{m m}
\end{array}\right]
$$

be the matrix generated by the correlation coefficients of the correlated $m$-dimensional Brownian motion $\left(B_{t}^{(1)}, \ldots, B_{t}^{(m)}\right)^{T}$ with

$$
\rho_{i k} \begin{cases}=1, & \text { if } i=k \\ <1, & \text { if } i \neq k\end{cases}
$$

The matrix $\Psi$ is nonsingular, symmetric, and positively semidefinite. So, there exists a nonsingular matrix $\bar{A}:=\left(\bar{a}_{i j}\right)_{m \times m}$ such that $\Psi=\bar{A} \bar{A}^{T}$. It can be shown that there exist $m$ independent Brownian motions $\widetilde{W}_{t}^{(1)}, \ldots, \widetilde{W}_{t}^{(m)}$ such that

$$
B_{t}^{(i)}=\sum_{j=1}^{m} \bar{a}_{i j} \widetilde{W}_{t}^{(j)}, \quad \forall i=1, \ldots, m .
$$

For details, refer to Exercise 4.16 in [13]. So far, we have derived a complete market with $m$ stocks and $m$-independent component Brownian motions. The $m$-stocks in the incomplete market can now be re-written as

$$
\mathrm{d} S_{t}^{(i)}=S_{t}^{(i)}\left(b_{i} \mathrm{~d} t+\left\|\sigma_{i}\right\| \sum_{j=1}^{m} \bar{a}_{i j} \mathrm{~d} \widetilde{W}_{t}^{(j)}\right), \quad \forall i=1, \ldots, m .
$$

The volatility matrix, denoted by $\widetilde{\sigma}$, under the completed market is given by

$$
\begin{aligned}
\widetilde{\sigma} & =\left[\begin{array}{cccc}
\left\|\sigma_{1}\right\| \bar{a}_{11} & \left\|\sigma_{1}\right\| \bar{a}_{12} & \cdots & \left\|\sigma_{1}\right\| \bar{a}_{1 m} \\
\left\|\sigma_{2}\right\| \bar{a}_{21} & \left\|\sigma_{2}\right\| \bar{a}_{22} & \cdots & \left\|\sigma_{2}\right\| \bar{a}_{2 m} \\
\vdots & \vdots & \ddots & \vdots \\
\left\|\sigma_{m}\right\| \bar{a}_{m 1} & \left\|\sigma_{m}\right\| \bar{a}_{m 2} & \cdots & \left\|\sigma_{m}\right\| \bar{a}_{m m}
\end{array}\right] \\
& =\left[\begin{array}{cccc}
\left\|\sigma_{1}\right\| & 0 & \cdots & 0 \\
0 & \left\|\sigma_{2}\right\| & \cdots & 0 \\
\vdots & \vdots & \ddots & \vdots \\
0 & 0 & \cdots & \left\|\sigma_{m}\right\|
\end{array}\right] \cdot \bar{A},
\end{aligned}
$$

Put

$$
\Sigma:=\left[\begin{array}{cccc}
\left\|\sigma_{1}\right\| & 0 & \cdots & 0 \\
0 & \left\|\sigma_{2}\right\| & \cdots & 0 \\
\vdots & \vdots & \ddots & \vdots \\
0 & 0 & \cdots & \left\|\sigma_{m}\right\|
\end{array}\right]
$$

Then

$$
\widetilde{\sigma}=\Sigma \bar{A} .
$$


With the help of $\widetilde{\sigma}$ and $\widetilde{W}_{t}=\left(\widetilde{W}_{t}^{(1)}, \ldots, \widetilde{W}_{t}^{(m)}\right)$, we transfer the model (7) to the following one in the completed market

$$
\begin{gathered}
\mathrm{d} X_{t}^{x_{0}, \pi}=\pi_{t}^{T}\left[b \mathrm{~d} t+\widetilde{\sigma} \mathrm{d} \widetilde{W}_{t}\right]+\left(X_{t}^{x_{0}, \pi}-\pi_{t}^{T} \mathbf{1}_{m}\right) r \mathrm{~d} t \\
+c \mathrm{~d} t-\mathrm{d} L_{t} \\
\quad X_{0}^{x_{0}, \pi}=x_{0},
\end{gathered}
$$

which is equivalent to

$$
\begin{aligned}
X_{t}^{x_{0}, \pi}= & \mathrm{e}^{r t}\left(x_{0}+\int_{0}^{t} \mathrm{e}^{-r s}\left(\pi_{s}^{T}\left(b-r \mathbf{1}_{m}\right)+c\right) \mathrm{d} s\right. \\
& \left.\quad+\int_{0}^{t} \mathrm{e}^{-r s} \pi_{s}^{T} \widetilde{\sigma} \mathrm{d} \widetilde{W}_{s}-\int_{0}^{t} \mathrm{e}^{-r s} \mathrm{~d} L_{s}\right) \\
= & \mathrm{e}^{r t} x_{0}+\frac{c\left(\mathrm{e}^{r t}-1\right)}{r}+\int_{0}^{t} \mathrm{e}^{r(t-s)}\left(\pi_{s}^{T}\left(b-r \mathbf{1}_{m}\right)\right) \mathrm{d} s \\
& +\int_{0}^{t} \mathrm{e}^{r(t-s)} \pi_{s}^{T} \widetilde{\sigma} \mathrm{d} \widetilde{W}_{s}-\int_{0}^{t} \mathrm{e}^{r(t-s)} \mathrm{d} L_{s} .
\end{aligned}
$$

Define

$$
\begin{aligned}
\widehat{X}_{t}^{x_{0}, \pi}= & \mathrm{e}^{-r t} X_{t}^{x_{0}, \pi} \\
= & x_{0}+\frac{c\left(1-\mathrm{e}^{-r t}\right)}{r}+\int_{0}^{t} \mathrm{e}^{-r s}\left(\pi_{s}^{T}\left(b-r \mathbf{1}_{m}\right)\right) \mathrm{d} s \\
& +\int_{0}^{t} \mathrm{e}^{-r s} \pi_{s}^{T} \widetilde{\sigma} \mathrm{d} \widetilde{W}_{s}-\int_{0}^{t} \mathrm{e}^{-r s} \mathrm{~d} L_{s}
\end{aligned}
$$

which represents the discounted value of $X_{t}^{x_{0}, \pi}$.

Suppose that the insurer has a utility function $U$ of his wealth and he/she aims to maximize the expected utility of his/her discounted terminal wealth, that is,

$$
\operatorname{maximize} \mathbb{E}\left[U\left(\widehat{X}_{T}^{x_{0}, \pi}\right)\right] \quad \text { over } \pi \in \Pi \text {. }
$$

The utility function $U$ is strictly concave and continuously differentiable on $(-\infty, \infty)$; thus there exists at most a unique optimal discounted terminal wealth for the company.

Furthermore, for any $\pi^{*} \in \Pi$ and $\pi \in \Pi$, we have

$$
\begin{aligned}
& \mathbb{E}\left[U\left(\widehat{X}_{T}^{x_{0}, \pi^{*}}\right)\right]-\mathbb{E}\left[U\left(\widehat{X}_{T}^{x_{0}, \pi}\right)\right] \\
& \quad \geq \mathbb{E}\left[U^{\prime}\left(\widehat{X}_{T}^{x_{0}, \pi^{*}}\right)\left(\widehat{X}_{T}^{x_{0}, \pi^{*}}-\widehat{X}_{T}^{x_{0}, \pi}\right)\right],
\end{aligned}
$$

from the concavity of $U$. according to (24) we claim the following proposition.

Proposition 2. If there exists a strategy $\pi^{*} \in \Pi$ such that

$$
\mathbb{E}\left[U^{\prime}\left(\widehat{X}_{T}^{x_{0}, \pi^{*}}\right) \widehat{X}_{T}^{x_{0}, \pi}\right] \quad \text { is constant over } \pi \in \Pi \text {, }
$$

then $\pi^{*}$ is the optimal trading strategy.
Remark 3. Such sufficient conditions to the optimal investment are well known in the martingale approach; refer to [9] and others.

By (22), (25) is equivalent to that

$$
\mathbb{E}\left[U^{\prime}\left(\widehat{X}_{T}^{x_{0}, \pi^{*}}\right) \int_{0}^{T}\left(\mathrm{e}^{-r s} \pi_{s}^{T}\left(b-r \mathbf{1}_{m}\right) \mathrm{d} s+\mathrm{e}^{-r s} \pi_{s}^{T} \widetilde{\sigma} \mathrm{d} \widetilde{W}_{s}\right)\right]
$$

is constant over $\pi \in \Pi$.

Thus, problem (23) can be solved by applying (26) in terms of the concrete utility functions.

It is well known that maximizing the expected quadratic utility is equivalent to finding a mean-variance efficient strategy while CARA utility is commonly used. Thus, we will work out the optimal strategies explicitly for these two utilities in the next section.

\section{Optimal Strategies for Different Utility Functions}

First, we introduce some notations and a martingale representation theorem that will be used. Let $\mathscr{P}$ be the predictable $\sigma$-algebra on $\Omega \times[0, T]$, which is generated by all leftcontinuous and $\left(\mathscr{F}_{t}\right)$-adapted processes, and $\widetilde{\mathscr{P}}:=\mathscr{P} \otimes$ $\mathscr{B}(\mathbb{R})$. Let $L(\mathscr{P})$ (resp., $L^{2}(\mathscr{P})$ ) be the set of all $\left(\mathscr{F}_{t}\right)$ predictable, $\mathbb{R}$-valued processes $\theta$ such that $\int_{0}^{T}|\theta(t)|^{2} \mathrm{~d} t<\infty$ a.s. (resp., $\left.\mathbb{E}\left[\int_{0}^{T}|\theta(t)|^{2} \mathrm{~d} t\right]<\infty\right)$. Let $L(\widetilde{\mathscr{P}})$ be the set of all $\widetilde{\mathscr{P}}_{-}$ measurable, $\mathbb{R}$-valued functions $\theta$ defined on $\Omega \times[0, T] \times \mathbb{R}$ such that

$$
\sqrt{\sum_{0<s \leq t}\left|\theta\left(s, \Delta L_{s}\right)\right|^{2} I_{\left\{\Delta L_{s} \neq 0\right\}}}
$$

is locally integrable and increasing, and also $\int_{\mathbb{R}}|\theta(t, x)| m(\mathrm{~d} x)<\infty$ a.s. for all $t \in[0, T]$, where $I_{\{\ldots\}}$ is the indicator function. Moreover, let $L^{2}(\widetilde{\mathscr{P}})$ be the set of all $\widetilde{\mathscr{P}}$-measurable, $\mathbb{R}$-valued functions $\theta$ defined on $\Omega \times[0, T] \times \mathbb{R}$ such that $\mathbb{E}\left[\int_{0}^{T} \int_{\mathbb{R}}|\theta(t, x)|^{2} m(\mathrm{~d} x) \mathrm{d} t\right]<\infty$. Finally, $L_{\mathscr{F}}^{2}$ denotes the set of all $\left(\mathscr{F}_{t}\right)$-adapted processes $\left(X_{t}\right)$ with Càdlàg paths such that $\mathbb{E}\left[\sup _{0 \leq t \leq T}\left|X_{t}\right|^{2}\right]<\infty$. It is well known that every square-integrable martingale belongs to $L_{\mathscr{F}}^{2}$, and it is easy to see that if $\pi \in \Pi$ then $\widehat{X}^{x_{0}, \pi} \in L_{\mathscr{F}}^{2}$.

One can consult Proposition 9.4 in [12] for the following result.

Lemma 4 (Martingale Representation). For any local (resp., square-integrable) martingale $\left(Z_{t}\right)$, there exist some $\theta_{1}=\left(\theta_{1}^{(1)}, \ldots, \theta_{1}^{(m)}\right)^{T}$ with $\theta_{1}^{T} \in \underbrace{L(\mathscr{P}) \times \cdots \times L(\mathscr{P})}_{m}$, 
and $\theta_{2} \in L(\widetilde{\mathscr{P}})($ resp., $\theta_{1}^{T} \in \underbrace{L^{2}(\mathscr{P}) \times \cdots \times L^{2}(\mathscr{P})}_{m}$, and $\theta_{2} \epsilon$ $\left.L^{2}(\widetilde{\mathscr{P}})\right)$ such that

$$
\begin{aligned}
Z_{t}= & Z_{0}+\int_{0}^{t}\left[\theta_{1}(s)\right]^{T} \mathrm{~d} \widetilde{W}_{s} \\
& +\int_{0}^{t} \int_{\mathbb{R}} \theta_{2}(s, x)(\mu(\mathrm{d} s, \mathrm{~d} x)-\nu(\mathrm{d} s, \mathrm{~d} x)),
\end{aligned}
$$

for all $t \in[0, T]$.

4.1. Quadratic Utility. In this subsection, we consider problem (23) for the quadratic utility function $U(x)=x-(\gamma / 2) x^{2}$, where $\gamma>0$ is a parameter. In case that there is only one stock and one bond in a complete market, a closed-form solution is obtained by Wang et al. [7]. Motivated by their work, we show that a closed-form solution can also be obtained when there are several stocks in an incomplete market. Since $U^{\prime}(x)=$ $1-\gamma x$, condition (26) can be written as

$$
\mathbb{E}\left[\left(1-\gamma \widehat{X}_{T}^{x_{0}, \pi^{*}}\right) \int_{0}^{T}\left(\mathrm{e}^{-r s} \pi_{s}^{T}\left(b-r \mathbf{1}_{m}\right) \mathrm{d} s+\mathrm{e}^{-r s} \pi_{s}^{T} \widetilde{\sigma} \mathrm{d} \widetilde{W}_{s}\right)\right]
$$

is constant over $\pi \in \Pi$.

Put

$$
Z_{t}^{*}=\mathbb{E}\left[1-\gamma \widehat{X}_{T}^{x_{0}, \pi^{*}} \mid \mathscr{F}_{t}\right], \quad t \in[0, T]
$$

Then $Z_{T}^{*}=1-\gamma \widehat{X}_{T}^{x_{0}, \pi^{*}}$ and $Z_{\tau}^{*}=\mathbb{E}\left[Z_{T}^{*} \mid \mathscr{F}_{\tau}\right]$ a.s. for any stopping time $\tau \leq T$ a.s.

Lemma 5. Let $\pi^{*} \in \Pi$. Then $\pi^{*}$ satisfies condition (29) if and only if there exists a $\theta_{2}^{*} \in L^{2}(\widetilde{\mathscr{P}})$ such that $\left(\widehat{X}^{x_{0}, \pi^{*}}, \pi^{*}, Z^{*}, \theta_{2}^{*}\right)$ solves the following forward-backward stochastic differential equation (FBSDE)

$$
\begin{aligned}
\mathrm{d} \widehat{X}_{t}= & \mathrm{e}^{-r t} \pi_{t}^{T}\left(b-r \mathbf{1}_{m}\right) \mathrm{d} t+\mathrm{e}^{-r t} \pi_{t}^{T} \widetilde{\sigma} \mathrm{d} \widetilde{W}_{t}+c \mathrm{e}^{-r t} \mathrm{~d} t \\
& -\mathrm{e}^{-r t} d L_{t}, \\
\widehat{X}_{0}=x_{0}, & \\
\mathrm{~d} Z_{t}= & -Z_{t-}\left(b-r \mathbf{1}_{m}\right)^{T}\left(\widetilde{\sigma}^{-1}\right)^{T} \mathrm{~d} \widetilde{W}_{t} \\
& +\mathrm{d}\left(\int_{0}^{t} \int_{\mathbb{R}} \theta_{2}(s, x)(\mu(\mathrm{d} s, \mathrm{~d} x)-v(\mathrm{~d} s, \mathrm{~d} x))\right), \\
Z_{T}=1 & -\gamma \widehat{X}_{T},
\end{aligned}
$$

for $\left(\widehat{X}, \pi, Z, \theta_{2}\right) \in L_{\mathscr{F}}^{2} \times \Pi \times L_{\mathscr{F}}^{2} \times L^{2}(\widetilde{\mathscr{P}})$.

Proof. Suppose $\pi^{*}$ satisfies condition (29). From (22) we have $\left(\widehat{X}^{x_{0}, \pi^{*}}, \pi^{*}\right) \in L_{\mathscr{F}}^{2} \times \Pi$ solving the forward SDE in (31) for $(\widehat{X}, \pi)$ and it is clear that $\left(Z_{t}^{*}\right)$ is a square-integrable martingale. For any stopping time $\tau \leq T$, let $\pi_{t}^{\tau, i}=$ $\left(0, \ldots, 0, I_{[t \leq \tau]}, 0, \ldots, 0,\right)^{T}, i=1, \ldots, m$, whose $i$ th component is $I_{[t \leq \tau]}$ and the other components are all 0 . Then $\pi^{\tau, i} \in$ $\Pi, i=1, \ldots, m$. Denote by $\widetilde{\sigma}_{i}$ the $i$ th row vector of $\widetilde{\sigma}$ for $i=1, \ldots, m$; that is, $\widetilde{\sigma}_{i}:=\left(\widetilde{\sigma}_{i 1}, \ldots, \widetilde{\sigma}_{i m}\right)$. For any positive integer $1 \leq i \leq m$, substituting $\pi^{\tau, i}$ into (29), we have that

$$
\begin{aligned}
\mathbb{E} & {\left[Z_{T}^{*} \int_{0}^{\tau}\left(\mathrm{e}^{-r s}\left(b_{i}-r\right) \mathrm{d} s+\mathrm{e}^{-r s} \widetilde{\sigma}_{i} \mathrm{~d} \widetilde{W}_{s}\right)\right] } \\
& =\mathbb{E}\left[\mathbb{E}\left[Z_{T}^{*} \mid \mathscr{F}_{\tau}\right] \int_{0}^{\tau}\left(\mathrm{e}^{-r s}\left(b_{i}-r\right) \mathrm{d} s+\mathrm{e}^{-r s} \widetilde{\sigma}_{i} \mathrm{~d} \widetilde{W}_{s}\right)\right] \\
& =\mathbb{E}\left[Z_{\tau}^{*} \int_{0}^{\tau}\left(\mathrm{e}^{-r s}\left(b_{i}-r\right) \mathrm{d} s+\mathrm{e}^{-r s} \widetilde{\sigma}_{i} \mathrm{~d} \widetilde{W}_{s}\right)\right]
\end{aligned}
$$

is constant over all stopping times $\tau \leq T$ a.s., which implies that

$$
\begin{array}{r}
Z_{t}^{*} \int_{0}^{t}\left(\mathrm{e}^{-r s}\left(b_{i}-r\right) \mathrm{d} s+\mathrm{e}^{-r s} \widetilde{\sigma}_{i} \mathrm{~d} \widetilde{W}_{s}\right) \quad \text { is a martingale, } \\
i=1, \ldots, m .
\end{array}
$$

Since $\left(Z_{t}^{*}\right)$ is a square-integrable martingale, by Lemma 4 , there are $\theta_{1}^{*}=\left(\theta_{1}^{(1), *}, \ldots, \theta_{1}^{(m), *}\right)^{T}$ with $\left(\theta_{1}^{*}\right)^{T} \in \underbrace{L^{2}(\mathscr{P}) \times \cdots \times L^{2}(\mathscr{P})}_{m}$ and $\theta_{2}^{*} \in L^{2}(\widetilde{P})$ such that

$$
\begin{aligned}
\mathrm{d} Z_{t}^{*}= & {\left[\theta_{1}^{*}(t)\right]^{T} \mathrm{~d} \widetilde{W}_{t} } \\
& +\mathrm{d}\left(\int_{0}^{t} \int_{\mathbb{R}} \theta_{2}^{*}(s, x)(\mu(\mathrm{d} s, \mathrm{~d} x)-\nu(\mathrm{d} s, \mathrm{~d} x))\right) .
\end{aligned}
$$

By Itô’s formula,

$$
\begin{aligned}
\mathrm{d} & \left(Z_{t}^{*} \int_{0}^{t}\left(\mathrm{e}^{-r s}\left(b_{i}-r\right) \mathrm{d} s+\mathrm{e}^{-r s} \widetilde{\sigma}_{i} \mathrm{~d} \widetilde{W}_{s}\right)\right) \\
& =\left(\left(b_{i}-r\right) Z_{t-}^{*}+\widetilde{\sigma}_{i} \theta_{1}^{*}(t)\right) \mathrm{e}^{-r t} \mathrm{~d} t+\text { a local martingale, }
\end{aligned}
$$

which together with (33) implies $\left(b_{i}-r\right) Z_{t-}^{*}+\widetilde{\sigma}_{i} \theta_{1}^{*}(t)=0$ for each $i=1, \ldots, m$. Thus, it is easy to see that

$$
\widetilde{\sigma} \theta_{1}^{*}(t)=-\left(b-r \mathbf{1}_{m}\right) Z_{t-}^{*},
$$

that is,

$$
\theta_{1}^{*}(t)=-\widetilde{\sigma}^{-1}\left(b-r \mathbf{1}_{m}\right) Z_{t-}^{*} .
$$

By (34), $\left(Z^{*}, \theta_{2}^{*}\right)$ solves the backward SDE in (31) for $\left(Z, \theta_{2}\right)$, and hence $\left(\widehat{X}^{x_{0}, \pi^{*}}, \pi^{*}, Z^{*}, \theta_{2}^{*}\right)$ solves $\operatorname{FBSDE}(31)$.

Conversely, suppose that there exists $\left(Z^{*}, \theta_{2}^{*}\right) \in L_{\mathscr{F}}^{2} \times$ $L^{2}(\widetilde{\mathscr{P}})$ such that $\left(\widehat{X}^{x_{0}, \pi^{*}}, \pi^{*}, Z^{*}, \theta_{2}^{*}\right)$ solves FBSDE (31). It is easy to verify that for any $\pi \in \Pi$, by Itô's formula, $\left(Z_{t}^{*} M_{t}^{\pi}\right)$ is a local martingale, where

$$
M_{t}^{\pi}:=\int_{0}^{t} \mathrm{e}^{-r s} \pi_{s}^{T}\left(b-r \mathbf{1}_{m}\right) \mathrm{d} s+\mathrm{e}^{-r s} \pi_{s}^{T} \widetilde{\sigma} \mathrm{d} \widetilde{W}_{s} .
$$


Furthermore, for any $\pi \in \Pi$, we have $M^{\pi} \in L_{\mathscr{F}}^{2}$. Hence,

$$
\begin{gathered}
\mathbb{E}\left[\sup _{0 \leq t \leq T}\left|Z_{t}^{*} M_{t}^{\pi}\right|\right] \\
\leq \sqrt{\mathbb{E}\left[\sup _{0 \leq t \leq T}\left|Z_{t}^{*}\right|^{2}\right] \cdot \mathbb{E}\left[\sup _{0 \leq t \leq T}\left|M_{t}^{\pi}\right|^{2}\right]}<\infty,
\end{gathered}
$$

which implies that the family

$$
\left\{Z_{\tau}^{*} M_{\tau}^{\pi}: \tau \text { is a stopping time and } \tau \leq T\right\}
$$

is uniformly integrable and hence $Z^{*} M^{\pi}$ is a martingale. Thus we have $\mathbb{E}\left[Z_{T}^{*} M_{T}^{\pi}\right]=0$ for any $\pi \in \Pi$, implying that $\pi^{*}$ satisfies condition (29).

In what follows, we will solve FBSDE (31) by two steps.

Step 1. Conjecturing the form of solution.

Put

$$
A_{t}=\exp \left\{\int_{0}^{t} a_{s} \mathrm{~d} s\right\}, \quad t \in[0, T]
$$

where $\left(a_{t}\right)$ is a nonrandom Lebesgue-integrable function to be determined. If $\left(\widehat{X}, \pi, Z, \theta_{2}\right)$ solves $\operatorname{FBSDE}(31)$, then by Itô's formula,

$$
\begin{aligned}
A_{T} Z_{T}= & Z_{0}+\int_{0}^{T} A_{s} \mathrm{~d} Z_{s}+\int_{0}^{T} Z_{s-} \mathrm{d} A_{s} \\
= & Z_{0}-\int_{0}^{T} A_{s} Z_{s-}\left(b-r \mathbf{1}_{m}\right)^{T}\left(\widetilde{\sigma}^{-1}\right)^{T} \mathrm{~d} \widetilde{W}_{s} \\
& +\int_{0}^{T} \int_{\mathbb{R}} A_{s} \theta_{2}(s, x)(\mu(\mathrm{d} s, \mathrm{~d} x)-\nu(\mathrm{d} s, \mathrm{~d} x)) \\
& +\int_{0}^{T} Z_{s^{-}} A_{s} a_{s} \mathrm{~d} s,
\end{aligned}
$$

which implies

$$
\begin{aligned}
\frac{1-Z_{T}}{\gamma} & \\
= & \frac{1}{\gamma}-\frac{1}{\gamma A_{T}} A_{T} Z_{T} \\
= & \frac{1}{\gamma}-\frac{Z_{0}}{\gamma A_{T}}+\frac{1}{\gamma A_{T}} \int_{0}^{T} A_{s} Z_{s^{-}}\left(b-r \mathbf{1}_{m}\right)^{T}\left(\tilde{\sigma}^{-1}\right)^{T} \mathrm{~d} \widetilde{W}_{s} \\
& -\frac{1}{\gamma A_{T}} \int_{0}^{T} \int_{\mathbb{R}} A_{s} \theta_{2}(s, x)(\mu(\mathrm{d} s, \mathrm{~d} x)-\nu(\mathrm{d} s, \mathrm{~d} x)) \\
& -\frac{1}{\gamma A_{T}} \int_{0}^{T} Z_{s^{-}} A_{s} a_{s} \mathrm{~d} s .
\end{aligned}
$$

Let $\left(\widehat{X}, \pi, Z, \theta_{2}\right)$ solve $\operatorname{FBSDE}(31)$. Then $\widehat{X}_{T}=\left(1-Z_{T}\right) / \gamma$. Comparing $\mathrm{d} \widetilde{W}_{t}$-term $\mathrm{d}(\mu-\nu)$-term, respectively, in (43) with those in (22) and noting (6), it is reasonable to conjecture

$$
\begin{gathered}
\frac{A_{t} Z_{t-}}{\gamma A_{T}} \tilde{\sigma}^{-1}\left(b-r \mathbf{1}_{m}\right)=\mathrm{e}^{-r t} \widetilde{\sigma}^{T} \pi_{t}, \\
\frac{A_{t}}{\gamma A_{T}} \theta_{2}(t, x)=\mathrm{e}^{-r t} x,
\end{gathered}
$$

that is,

$$
\begin{gathered}
\pi_{t}=\frac{A_{t} Z_{t-} \mathrm{e}^{r t}}{\gamma A_{T}}\left(\widetilde{\sigma}^{T}\right)^{-1} \tilde{\sigma}^{-1}\left(b-r \mathbf{1}_{m}\right), \\
\theta_{2}(t, x)=\frac{\gamma \mathrm{e}^{-r t} x A_{T}}{A_{t}} .
\end{gathered}
$$

Note that by (19), $\Sigma$ and $A$ are both nonsingular, $\left(\widetilde{\sigma}^{T}\right)^{-1} \tilde{\sigma}^{-1}=$ $\left(\widetilde{\sigma} \widetilde{\sigma}^{T}\right)^{-1}=\left(\Sigma \bar{A} \bar{A}^{T} \Sigma^{T}\right)^{-1}=(\Sigma \Psi \Sigma)^{-1}$. Hence, $(45)$ can be rewritten as

$$
\begin{gathered}
\pi_{t}=\frac{A_{t} Z_{t-} \mathrm{e}^{r t}}{\gamma A_{T}}(\Sigma \Psi \Sigma)^{-1}\left(b-r \mathbf{1}_{m}\right), \\
\theta_{2}(t, x)=\frac{\gamma \mathrm{e}^{-r t} x A_{T}}{A_{t}} .
\end{gathered}
$$

Substituting (44) and (46) into (43), we have

$$
\begin{aligned}
\frac{1-Z_{T}}{\gamma} & \frac{1}{\gamma}-\frac{Z_{0}}{\gamma A_{T}}+\int_{0}^{T} \mathrm{e}^{-r s} \pi_{s}^{T} \widetilde{\sigma} \mathrm{d} \widetilde{W}_{s}-\int_{0}^{T} \mathrm{e}^{-r s} \mathrm{~d} L_{s} \\
& -\frac{1}{\gamma A_{T}} \int_{0}^{T} Z_{s-} A_{s} a_{s} \mathrm{~d} s \\
= & \frac{1}{\gamma}-\frac{Z_{0}}{\gamma A_{T}}+\widehat{X}_{T}-x_{0}-\frac{c\left(1-\mathrm{e}^{-r T}\right)}{r} \\
& -\int_{0}^{T} \mathrm{e}^{-r s} \pi_{s}^{T}\left(b-r \mathbf{1}_{m}\right) \mathrm{d} s-\frac{1}{\gamma A_{T}} \int_{0}^{T} Z_{s-} A_{s} a_{s} \mathrm{~d} s \\
= & \widehat{X}_{T}+\frac{1}{\gamma}-\frac{Z_{0}}{\gamma A_{T}}-x_{0}-\frac{c\left(1-\mathrm{e}^{-r T}\right)}{r} \\
& -\int_{0}^{T} \frac{A_{s} Z_{s-}}{\gamma A_{T}}\left(b-r \mathbf{1}_{m}\right)^{T}(\Sigma \Psi \Sigma)^{-1}\left(b-r \mathbf{1}_{m}\right) \mathrm{d} s \\
& -\frac{1}{\gamma A_{T}} \int_{0}^{T} Z_{s-} A_{s} a_{s} \mathrm{~d} s,
\end{aligned}
$$


where the second equality follows from the forward SDE in (31) and the third equality holds owing to $\left[(\Sigma \Psi \Sigma)^{-1}\right]^{T}=$ $(\Sigma \Psi \Sigma)^{-1}$. If we take

$$
\begin{aligned}
a_{t} & =-\left(b-r \mathbf{1}_{m}\right)^{T}(\Sigma \Psi \Sigma)^{-1}\left(b-r \mathbf{1}_{m}\right), \\
Z_{0} & =A_{T}-\gamma A_{T} x_{0}-\gamma A_{T} \frac{c\left(1-\mathrm{e}^{-r T}\right)}{r} \\
& =\left(1-\gamma x_{0}-\frac{\gamma c\left(1-\mathrm{e}^{-r T}\right)}{r}\right) \mathrm{e}^{-\left(b-r \mathbf{1}_{m}\right)^{T}(\Sigma \Psi \Sigma)^{-1}\left(b-r \mathbf{1}_{m}\right) T},
\end{aligned}
$$

then (47) is reduced as

$$
\frac{1-Z_{T}}{\gamma}=\widehat{X}_{T}
$$

and FBSDE (31) is solved.

Step 2. Verifying the conjecture in Step 1.

Let

$$
\begin{aligned}
& \theta_{2}^{*}(t, x) \\
& \quad=\gamma \exp \left\{-r t-\left(b-r \mathbf{1}_{m}\right)^{T}(\Sigma \Psi \Sigma)^{-1}\left(b-r \mathbf{1}_{m}\right)(T-t)\right\} x,
\end{aligned}
$$

$$
Z_{0}^{*}=\left(1-\gamma x_{0}-\frac{\gamma c\left(1-\mathrm{e}^{-r T}\right)}{r}\right) \mathrm{e}^{-\left(b-r \mathbf{1}_{m}\right)^{T}(\Sigma \Psi \Sigma)^{-1}\left(b-r \mathbf{1}_{m}\right) T}
$$

Then the following SDE

$$
\begin{aligned}
\mathrm{d} Z_{t}= & -Z_{t-}\left(b-r \mathbf{1}_{m}\right)^{T}\left(\widetilde{\sigma}^{-1}\right)^{T} \mathrm{~d} \widetilde{W}_{t} \\
& +\mathrm{d}\left(\int_{0}^{t} \int_{\mathbb{R}} \theta_{2}^{*}(s, x)(\mu(\mathrm{d} s, \mathrm{~d} x)-\nu(\mathrm{d} s, \mathrm{~d} x))\right)
\end{aligned}
$$

has a solution of the form

$$
\begin{aligned}
& Z_{t}^{*} \\
& =M_{t}\left(Z_{0}^{*}+\int_{0}^{t} \int_{\mathbb{R}} M_{s}^{-1} \theta_{2}^{*}(s, x)(\mu(\mathrm{d} s, \mathrm{~d} x)-\nu(\mathrm{d} s, \mathrm{~d} x))\right) \\
& =M_{t}\left(Z_{0}^{*}+\gamma \int_{0}^{t} M_{s}^{-1} \exp \left\{-r s-\left(b-r \mathbf{1}_{m}\right)^{T}(\Sigma \Psi \Sigma)^{-1}\right.\right. \\
& \left.\left.\times\left(b-r \mathbf{1}_{m}\right)(T-s)\right\} \mathrm{d} L_{s}\right),
\end{aligned}
$$

where

$$
\begin{aligned}
M_{t}=\exp \{ & -\left(b-r \mathbf{1}_{m}\right)^{T}\left(\widetilde{\sigma}^{-1}\right)^{T} \widetilde{W}_{t} \\
& \left.-\frac{1}{2}\left(b-r \mathbf{1}_{m}\right)^{T}(\Sigma \Psi \Sigma)^{-1}\left(b-r \mathbf{1}_{m}\right) t\right\} .
\end{aligned}
$$

Furthermore, let

$$
\begin{aligned}
\pi_{t}^{*}= & \frac{1}{\gamma} \exp \left\{\left(b-r \mathbf{1}_{m}\right)^{T}(\Sigma \Psi \Sigma)^{-1}\left(b-r \mathbf{1}_{m}\right)(T-t)+r t\right\} \\
& \times Z_{t-}^{*}(\Sigma \Psi \Sigma)^{-1}\left(b-r \mathbf{1}_{m}\right),
\end{aligned}
$$

and $\widehat{X}_{T}^{x_{0}, \pi^{*}}$ is obtained through the forward SDE in (31). Then by the procedure same as that in (47), it is easy to verify that

$$
\frac{1-Z_{T}^{*}}{\gamma}=\widehat{X}_{T}^{x_{0}, \pi^{*}}, \quad \text { that is, } 1-\gamma \widehat{X}_{T}^{x_{0}, \pi^{*}}=Z_{T}^{*} .
$$

Thus, $\left(\widehat{X}^{x_{0}, \pi^{*}}, \pi^{*}, Z^{*}, \theta_{2}^{*}\right)$ solves FBSDE (31).

Finally, by Proposition 2, Lemma 5, and arguments in Steps 1-2, we have the following theorem.

Theorem 6. Let $\pi^{*}$ be defined as in (51)-(55). Then $\pi^{*}$ is the optimal trading strategy of problem (23) for the quadratic utility function $U(x)=x-(\gamma / 2) x^{2}$.

4.2. CARA Utility. In this subsection, we consider problem (23) for the CARA utility function $U(x)=-(1 / \gamma) \mathrm{e}^{-\gamma x}$, where $\gamma>0$. The procedure is similar to what is done in [7]. Since $U^{\prime}(x)=\mathrm{e}^{-\gamma x}$, condition (26) can be written as

$$
\mathbb{E}\left[\mathrm{e}^{-\gamma \widehat{X}_{T}^{x_{0}, \pi^{*}}} \int_{0}^{T}\left(\mathrm{e}^{-r s} \pi_{s}^{T}\left(b-r \mathbf{1}_{m}\right) \mathrm{d} s+\mathrm{e}^{-r s} \pi_{s}^{T} \widetilde{\sigma} \mathrm{d} \widetilde{W}_{s}\right)\right]
$$

is constant over $\pi \in \Pi$.

Similar to the case of quadratic utility, in what follows, we split the procedure into three steps.

Step 1. Conjecturing the form of $\pi^{*}$ that satisfies condition (57).

Put

$$
Z_{T}^{*}:=\frac{\mathrm{e}^{-\gamma \widehat{X}_{T}^{x_{0}, \pi^{*}}}}{\mathbb{E}\left[\mathrm{e}^{-\gamma \widehat{X}_{T}^{x_{0}, \pi^{*}}}\right]},
$$

and $Z_{t}^{*}:=\mathbb{E}\left[Z_{T}^{*} \mid \mathscr{F}_{t}\right]$ for all $t \in[0, T]$. Then $Z_{\tau}^{*}:=\mathbb{E}\left[Z_{T}^{*} \mid\right.$ $\left.\mathscr{F}_{t}\right]$ a.s. for any stopping time $\tau \leq T$ a.s. Let $\mathbb{Q}$ be a probability measure on $(\Omega, \mathscr{F})$ such that $\mathrm{d} Q / \mathrm{d} P=Z_{T}^{*}$.

For any stopping time $\tau \leq T$ a.s., let $\pi_{t}^{\tau, i}=$ $\left(0, \ldots, 0, I_{[t \leq \tau]}, 0, \ldots, 0,\right)^{T}, i=1, \ldots, m$, whose $i$ th component is $I_{[t<\tau]}$ and the other components are all 0 . Then $\pi^{\tau, i} \in$ $\Pi, i=1, \ldots, m$. As in Section 4.1, denote by $\widetilde{\sigma}_{i}$ the $i$ th row vector of $\tilde{\sigma}$ for $i=1, \ldots, m$; that is, $\widetilde{\sigma}_{i}:=\left(\widetilde{\sigma}_{i 1}, \ldots, \widetilde{\sigma}_{i m}\right)$. For any positive integer $1 \leq i \leq m$, substituting $\pi^{\tau, i}$ into (57), we have

$$
\begin{aligned}
\mathbb{E}\left[Z_{T}^{*} \int_{0}^{\tau}\left(\mathrm{e}^{-r s}\left(b_{i}-r\right) \mathrm{d} s+\mathrm{e}^{-r s} \widetilde{\sigma}_{i} \mathrm{~d} \widetilde{W}_{s}\right)\right] \\
\quad=\mathbb{E}_{\mathbb{Q}}\left[\int_{0}^{\tau} \mathrm{e}^{-r s}\left(b_{i}-r\right) \mathrm{d} s+\mathrm{e}^{-r s} \widetilde{\sigma}_{i} \mathrm{~d} \widetilde{W}_{s}\right]
\end{aligned}
$$


being constant over all stopping times $\tau \leq T$ a.s., which implies that

$$
\int_{0}^{t} \mathrm{e}^{-r s}\left(b_{i}-r\right) \mathrm{d} s+\mathrm{e}^{-r s} \widetilde{\sigma}_{i} \mathrm{~d} \widetilde{W}_{s} \text { is a martingale under } \mathbb{Q}
$$$$
i=1, \ldots, m \text {. }
$$

Since $\left(Z_{t}^{*}\right)$ is a martingale, then

$$
K_{t}:=\int_{0}^{t} \frac{1}{Z_{s-}^{*}} \mathrm{~d} Z_{s}^{*}, \quad t \in[0, T]
$$

is a local martingale. By Lemma 4 , there exist some $\theta_{1}=$ $\left(\theta_{1}^{(1)}, \ldots, \theta_{1}^{(m)}\right)^{T}$ satisfying $\theta_{1}^{T} \in \underbrace{L(\mathscr{P}) \times \cdots \times L(\mathscr{P})}_{m}$ and $\theta_{2} \in$ $L(\widetilde{\mathscr{P}})$ such that

$$
\begin{aligned}
\mathrm{d} K_{t}= & \left(\theta_{1}(t)\right)^{T} \mathrm{~d} \widetilde{W}_{t} \\
& +\mathrm{d}\left(\int_{0}^{t} \int_{\mathbb{R}} \theta_{2}(s, x)(\mu(\mathrm{d} s, \mathrm{~d} x)-\nu(\mathrm{d} s, \mathrm{~d} x))\right),
\end{aligned}
$$

that is,

$$
\begin{aligned}
\mathrm{d} Z_{t}^{*}=Z_{t-}^{*} & {\left[\left(\theta_{1}(t)\right)^{T} \mathrm{~d} \widetilde{W}_{t}\right.} \\
& \left.+\mathrm{d}\left(\int_{0}^{t} \int_{\mathbb{R}} \theta_{2}(s, x)(\mu(\mathrm{d} s, \mathrm{~d} x)-\nu(\mathrm{d} s, \mathrm{~d} x))\right)\right] .
\end{aligned}
$$

Furthermore, by the Doléans-Dade exponential formula, we have

$$
\begin{aligned}
Z_{t}^{*}=\exp & \left\{\int_{0}^{t}\left(\theta_{1}(s)\right)^{T} \mathrm{~d} \widetilde{W}_{s}-\frac{1}{2} \int_{0}^{t}\left(\theta_{1}(s)\right)^{T} \theta_{1}(s) \mathrm{d} s\right. \\
& +\int_{0}^{t} \int_{\mathbb{R}} \theta_{2}(s, x)(\mu(\mathrm{d} s, \mathrm{~d} x)-\nu(\mathrm{d} s, \mathrm{~d} x)) \\
& \left.+\int_{0}^{t} \int_{\mathbb{R}}\left(\log \left(1+\theta_{2}(s, x)\right)-\theta_{2}(s, x)\right) \mu(\mathrm{d} s, \mathrm{~d} x)\right\} .
\end{aligned}
$$

By Girsanov's Theorem, we know that $\widetilde{W}_{t}-\int_{0}^{t} \theta_{1}(s) \mathrm{d} s$ is an $m$ dimensional Brownian motion under $\mathbb{Q}$, which together with (60) implies $\widetilde{\sigma}_{i} \theta_{1}(t)=-\left(b_{i}-r\right), i=1, \ldots, m$. Thus, it is easy to see that

$$
\widetilde{\sigma} \theta_{1}(t)=-\left(b-r \mathbf{1}_{m}\right)
$$

that is,

$$
\theta_{1}(t)=-\widetilde{\sigma}^{-1}\left(b-r \mathbf{1}_{m}\right)
$$

On the other hand, by (22), we have

$$
\begin{aligned}
\mathrm{e}^{-\gamma \widehat{X}_{T}^{x_{0}, \pi^{*}}}=\exp \{ & -\gamma x_{0}-\frac{\gamma c\left(1-\mathrm{e}^{-r T}\right)}{r} \\
& -\gamma \int_{0}^{T} \mathrm{e}^{-r s}\left(\pi_{s}^{*}\right)^{T}\left(b-r \mathbf{1}_{m}\right) \mathrm{d} s \\
& \left.-\gamma \int_{0}^{T} \mathrm{e}^{-r s}\left(\pi_{s}^{*}\right)^{T} \widetilde{\sigma} \mathrm{d} \widetilde{W}_{s}+\gamma \int_{0}^{T} \mathrm{e}^{-r s} \mathrm{~d} L_{s}\right\} .
\end{aligned}
$$

In order to have (58), comparing $\mathrm{d} \widetilde{W}_{t}$-term and $\mathrm{d} \mu$-term, respectively, in (64) with those in (67) and taking (66) into account, it is reasonable to conjecture

$$
\begin{gathered}
-\widetilde{\sigma}^{-1}\left(b-r \mathbf{1}_{m}\right)=-\gamma \mathrm{e}^{-r t} \tilde{\sigma}^{T} \pi_{t}^{*}, \\
\log \left(1+\theta_{2}(t, x)\right)=\gamma \mathrm{e}^{-r t} x,
\end{gathered}
$$

that is,

$$
\begin{gathered}
\pi_{t}^{*}=\frac{\mathrm{e}^{r t}\left(\widetilde{\sigma}^{T}\right)^{-1} \tilde{\sigma}^{-1}\left(b-r \mathbf{1}_{m}\right)}{\gamma}=\frac{\mathrm{e}^{r t}(\Sigma \Psi \Sigma)^{-1}\left(b-r \mathbf{1}_{m}\right)}{\gamma} \\
\theta_{2}(t, x)=\mathrm{e}^{\gamma x \mathrm{e}^{-r t}}-1 .
\end{gathered}
$$

Step 2. Verifying that $Z_{T}^{*}$ in (64) satisfies (58), where $\pi^{*}$ and $\theta_{2}$ are defined in (69), and $\theta_{1}$ is defined in (66).

Substituting (69) into (67), we have

$$
\mathrm{e}^{-\gamma \widehat{X}_{T}^{x_{0}, \pi^{*}}}=I_{T} H_{T},
$$

where

$$
\begin{gathered}
I_{T}=\exp \left\{-\gamma x_{0}-\frac{\gamma c\left(1-\mathrm{e}^{-r T}\right)}{r}\right. \\
\left.-\left(b-r \mathbf{1}_{m}\right)^{T}(\Sigma \Psi \Sigma)^{-1}\left(b-r \mathbf{1}_{m}\right) T\right\}, \\
H_{T}=\exp \left\{-\left(b-r \mathbf{1}_{m}\right)^{T}\left(\widetilde{\sigma}^{T}\right)^{-1} \widetilde{W}_{T}+\gamma \int_{0}^{T} \mathrm{e}^{-r s} \mathrm{~d} L_{s}\right\} .
\end{gathered}
$$

For $\theta_{1}$ and $\theta_{2}$ defined as above, it is easy to see that $Z^{*}$ is a martingale. Substituting (66) and (69) into (64), we have

$$
Z_{T}^{*}=J_{T} H_{T}
$$

where

$$
J_{T}=\exp \left\{-\frac{\left(b-r \mathbf{1}_{m}\right)^{T}(\Sigma \Psi \Sigma)^{-1}\left(b-r \mathbf{1}_{m}\right)}{2}\right.
$$

$$
\left.+\int_{0}^{T} \int_{\mathbb{R}}\left(\gamma \mathrm{e}^{-r s} x-\mathrm{e}^{\gamma x \mathrm{e}^{-r s}}+1\right) \nu(\mathrm{d} s, \mathrm{~d} x T)\right\}
$$


is a constant. Since $Z^{*}$ is a martingale, we have $\mathbb{E}\left[Z_{T}^{*}\right]=1$ and hence

$$
\mathbb{E}\left[H_{T}\right]=J_{T}^{-1} .
$$

Finally, by (70)-(74), we have

$$
\frac{\mathrm{e}^{-\gamma \widehat{X}_{T}^{x_{0}, \pi^{*}}}}{\mathbb{E}\left[\mathrm{e}^{\left.-\gamma \widehat{X}_{T}^{x_{T}, \pi^{*}}\right]}\right.}=\frac{I_{T} H_{T}}{I_{T} \mathbb{E}\left[H_{T}\right]}=J_{T} H_{T}=Z_{T}^{*},
$$

which is just what we desired.

Step 3. Verifying that $\pi^{*}$ in (69) satisfies condition (57).

It is clear that $\mathbb{E}\left[\left(Z_{T}^{*}\right)^{2}\right]<\infty$. For any $\pi \in \Pi$, by Girsanov's Theorem, we can see that

$$
\begin{array}{r}
M_{t}^{\pi}:=\int_{0}^{t} \mathrm{e}^{-r s} \pi_{s}^{T}\left(b-r \mathbf{1}_{m}\right) \mathrm{d} s+\mathrm{e}^{-r s} \pi_{s}^{T} \widetilde{\sigma} \mathrm{d} \widetilde{W}_{s}, \\
t \in[0, T],
\end{array}
$$

is a local martingale under $\mathbb{Q}$. Furthermore, for any $\pi \in \Pi$, we have $M^{\pi} \in L_{\mathscr{F}}^{2}$. Hence

$$
\begin{aligned}
\mathbb{E}_{\mathbb{Q}}\left[\sup _{0 \leq t \leq T}\left|M_{t}^{\pi}\right|\right] & =\mathbb{E}\left[Z_{T}^{*} \sup _{0 \leq t \leq T}\left|M_{t}^{\pi}\right|\right] \\
& \leq \sqrt{\mathbb{E}\left[\left(Z_{T}^{*}\right)^{2}\right] \cdot \mathbb{E}\left[\sup _{0 \leq t \leq T}\left|M_{t}^{\pi}\right|^{2}\right]}<\infty,
\end{aligned}
$$

which implies that the family

$$
\left\{M_{\tau}^{\pi}: \tau \text { is a stopping time and } \tau \leq T\right\}
$$

is uniformly integrable under $\mathbb{Q}$. Hence $M^{\pi}$ is a martingale under $\mathbb{Q}$. Thus we have $\mathbb{E}_{\mathbb{Q}}\left[M_{T}^{\pi}\right]=0$ for any $\pi \in \Pi$, which implies (57).

By Proposition 2 and arguments in steps 5-7, we can claim the following theorem.

Theorem 7. Let $\pi^{*}$ be defined as in (69). Then $\pi^{*}$ is the optimal trading strategy of problem (23) for the CARA utility function $U(x)=-(1 / \gamma) \mathrm{e}^{-\gamma x}$.

\section{Remarks and Computational Results}

In this section, we present some remarks and conduct computational experiments for the optimal investment problems of this paper according to the real market data. The objective of these computational tests is to contrast the insurer's optimal strategies in an incomplete market with those in a complete

\begin{tabular}{|c|c|c|}
\hline Industry & Company & Code \\
\hline Aerospace and defense & Honeywell Intl. & $\mathrm{HON}$ \\
\hline Automanufactures & Toyota Motor Corp. ADR & $\mathrm{TM}$ \\
\hline $\begin{array}{l}\text { Biotechnology and drug } \\
\text { manufacturers }\end{array}$ & Johnson \& Johnson & JNJ \\
\hline Chemicals & $\begin{array}{c}\text { EI DuPont de Nemours \& } \\
\text { Co. }\end{array}$ & DD \\
\hline $\begin{array}{l}\text { Communication } \\
\text { equipment }\end{array}$ & Qualcomm & QCOM \\
\hline Computer software & Microsoft & MSFT \\
\hline Discount & Wal-Mart Stores Inc. & WMT \\
\hline $\begin{array}{l}\text { Diversified computer } \\
\text { systems }\end{array}$ & Hewlett-Packard Co. & HPQ \\
\hline $\begin{array}{l}\text { Major integrated oil and } \\
\text { gas }\end{array}$ & BP Plc. & $\mathrm{BP}$ \\
\hline $\begin{array}{l}\text { Semiconductor-broad } \\
\text { line }\end{array}$ & Intel Corp. & INTC \\
\hline Telecom services & AT \& T & $\mathrm{T}$ \\
\hline $\begin{array}{l}\text { Utilities (gas and } \\
\text { electric) }\end{array}$ & Duke Energy Corporation & DUK \\
\hline
\end{tabular}
market.

According to Theorems 6 and 7, the optimal strategy of CARA utility is independent of the claim process, while that of quadratic utility is related to the claim process. This is due to the specific nature of the CARA utility.
TABLE 1: Stocks in a complete market.

5.1. Quadratic Utility. For quadratic utility, as shown in (51)(55), $\pi^{*}$ is a left-continuous process with a jump

$$
\pi_{t+}^{*}-\pi_{t}^{*}=(\Sigma \Psi \Sigma)^{-1}\left(b-r \mathbf{1}_{m}\right) \Delta L_{t},
$$

at any time $t$, where $\Delta L_{t}$ is the claim size at time $t$. From (79) the effect of claims on the efficient strategy of quadratic utility can be interpreted as follows.

(i) When no claim occurs at time $t$, the wealth process is continuous, and so is $\pi_{t}^{*}$.

(ii) When a claim occurs at time $t$, the wealth process has a jump $-\Delta L_{t}$, which is just the corresponding claim size; the optimal strategy also has a jump and the jump size on each stock is the corresponding element of $(\Sigma \Psi \Sigma)^{-1}\left(b-r \mathbf{1}_{m}\right) \Delta L_{t}$.

In terms of (79), we can consider the transaction cost of realizing the optimal strategy of quadratic utility. According to [14], the transaction cost is quantified by the portfolio weight turnover for a portfolio $\pi$, that is,

$$
\left\|\pi_{t+}-\pi_{t}\right\|_{1}
$$

where $\|\cdot\|_{1}$ denotes the 1-norm. Since transaction cost is an important aspect of any investment strategy, we are interested in comparing the costs of implementing the optimal strategies in an incomplete market with those in a complete market.

Suppose there are 12 underline Brownian motions, 12 stocks for investment in a complete market, and 10 stocks in an incomplete market. The universe of assets are chosen from the 12 industry categories of finance.cn.yahoo.com in 2009 (see Tables 1 and 2). The appreciation rate vector $b$ is estimated through the mean rate of return of each stock. The covariance matrix $a$ of the rate of return on 12 stocks is estimated based 
TABLE 2: Stocks in an incomplete market.

\begin{tabular}{|c|c|c|}
\hline Industry & Company & Code \\
\hline Aerospace and defense & Honeywell Intl. & $\mathrm{HON}$ \\
\hline Automanufactures & Toyota Motor Corp. ADR & $\mathrm{TM}$ \\
\hline $\begin{array}{l}\text { Biotechnology and drug } \\
\text { manufacturers }\end{array}$ & Johnson \& Johnson & JNJ \\
\hline Chemicals & $\begin{array}{c}\text { EI DuPont de Nemours \& } \\
\text { Co. }\end{array}$ & $\mathrm{DD}$ \\
\hline $\begin{array}{l}\text { Communication } \\
\text { equipment }\end{array}$ & Qualcomm & QCOM \\
\hline Computer software & Microsoft & MSFT \\
\hline $\begin{array}{l}\text { Diversified computer } \\
\text { systems }\end{array}$ & Hewlett-Packard Co. & HPQ \\
\hline $\begin{array}{l}\text { Major integrated oil \& } \\
\text { gas }\end{array}$ & BP Plc. & $\mathrm{BP}$ \\
\hline $\begin{array}{l}\text { Semiconductor-broad } \\
\text { line }\end{array}$ & Intel Corp. & INTC \\
\hline Utilities (gas \& electric) & Duke Energy Corporation & DUK \\
\hline
\end{tabular}

on the real market data. In a complete market, the volatility matrix $\widehat{\sigma}$ is calculated from $a$ such that $\widehat{\sigma} \widehat{\sigma}^{T}=a$. Next, the volatility matrix $\sigma$ of the 10 stocks in an incomplete market is obtained by eliminating corresponding row of $\widehat{\sigma}$. Let the riskfree rate $r=0.02$ and the claim size is distributed according to the exponential distribution with parameter 1 ; that is, $F(y)=$ $1-\mathrm{e}^{-y}$. Let $t=0,1 / 12,1 / 6, \ldots, 2$. With exponential claim size $\Delta L_{t}$ randomly generated and $\Psi, \Sigma$ calculated from (13) and (18), Figure 1 plots the transaction cost in an incomplete market and in a complete market, respectively.

We easily observe that the optimal strategies in an incomplete market incurs lower transaction cost than those in a complete market. Because there are fewer stocks in an incomplete market, than in a complete market, the number of stocks whose investment amount will be changed is smaller in an incomplete market. Thus the transaction cost for fewer stocks is low.

5.2. CARA Utility. For the optimal strategy of CARA utility, (69) shows that the discounted amount held in each stock is fixed. A similar investment strategy for CARA utility in an incomplete market is also obtained in Wang [11]. However, the expression of the optimal strategy in [11] contains a g-inverse of matrix $\sigma \sigma^{T}$, which is not unique. In our results, $(\Sigma \Psi \Sigma)^{-1}$ replaces the g-inverse matrix, which makes the optimal strategy explicit and deterministic. Due to the determinacy and nonrandomness of the optimal strategy for CARA utility, we can analyze the differences between complete market and incomplete market conveniently from the results of CARA utility.

The experimental procedure is the same as in Section 5.1 and all the parameters are set as in Section 5.1 except $t$. Let $T=$ 1 represent one year and the insurer rebalances his portfolio once a month. Then $t=0,1 / 12,1 / 6, \ldots, 11 / 12$ and there are 12 investment periods.

In Figure 2, we compare the average total dollar amount invested in stocks in an incomplete market with that in

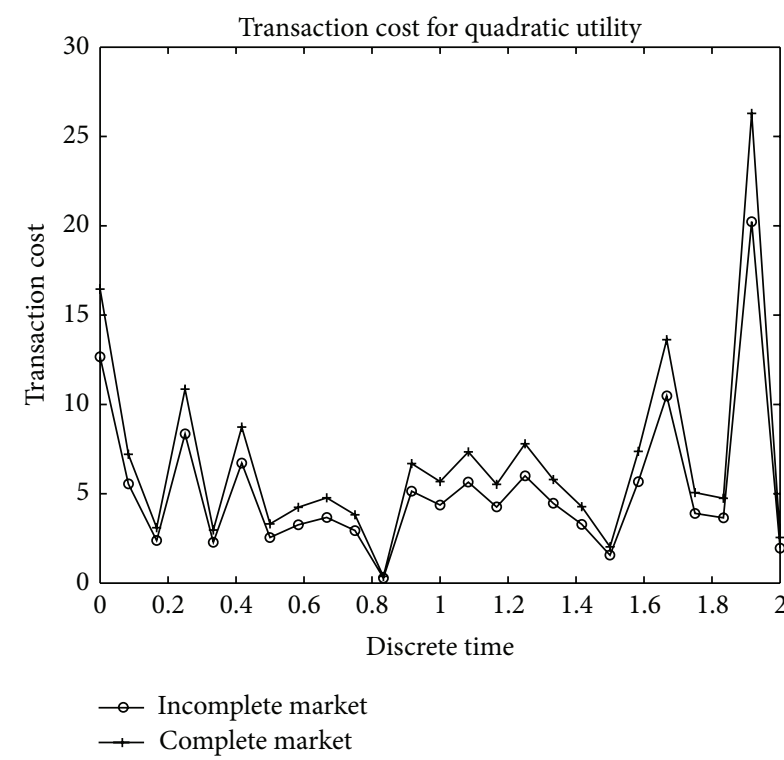

FIGURE 1: Transaction cost for quadratic utility.

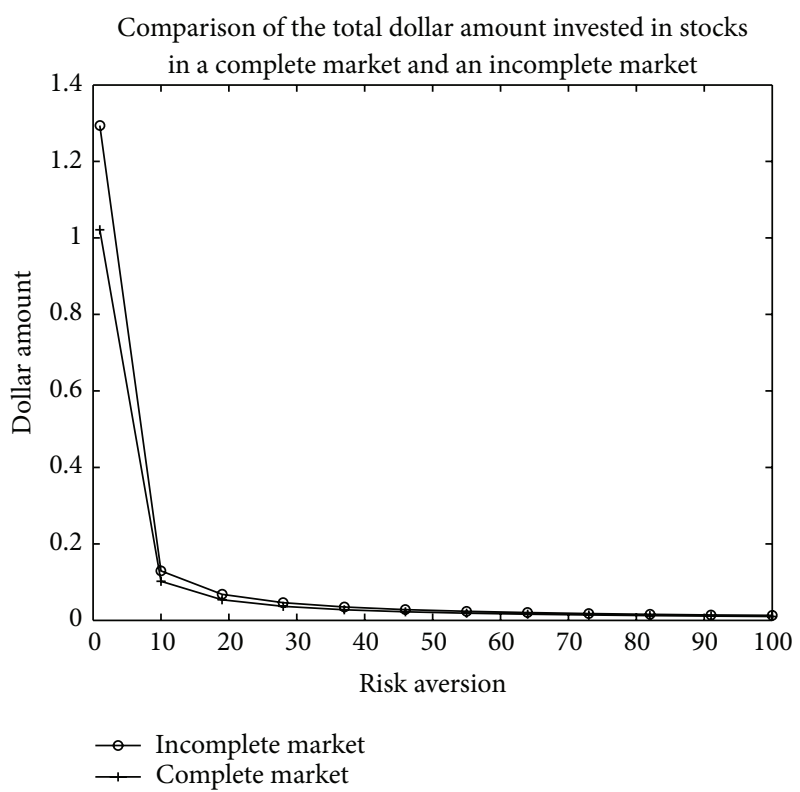

FIGURE 2: Comparison of the average total dollar amount invested in stocks in a complete market and an incomplete market.

a complete market as the risk aversion coefficient $\gamma$ ranges from 1 to 100. The average total dollar amount invested in stocks for a sequence of portfolios $\left\{\pi_{t}\right\}_{t=0}^{n}$ with $m$ stocks is defined as

$$
\bar{\pi}=\frac{1}{n+1} \sum_{t=0}^{n} \pi_{t}^{T} \mathbf{1}_{m}
$$

From Figure 2 we find that in an incomplete market, the insurer will invest more money in stocks (risky assets). The practical number of stocks in an incomplete market is less than the ideal number of stocks. For example, in our experiments, there is only 10 stocks for investment while 


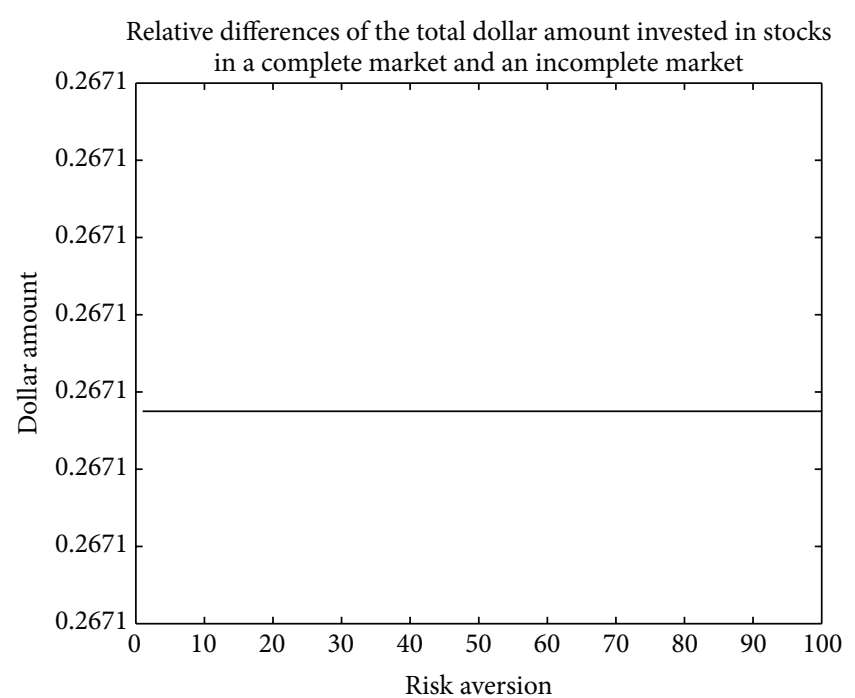

FIgURE 3: Relative differences of the average total dollar amount invested in stocks between the two markets.

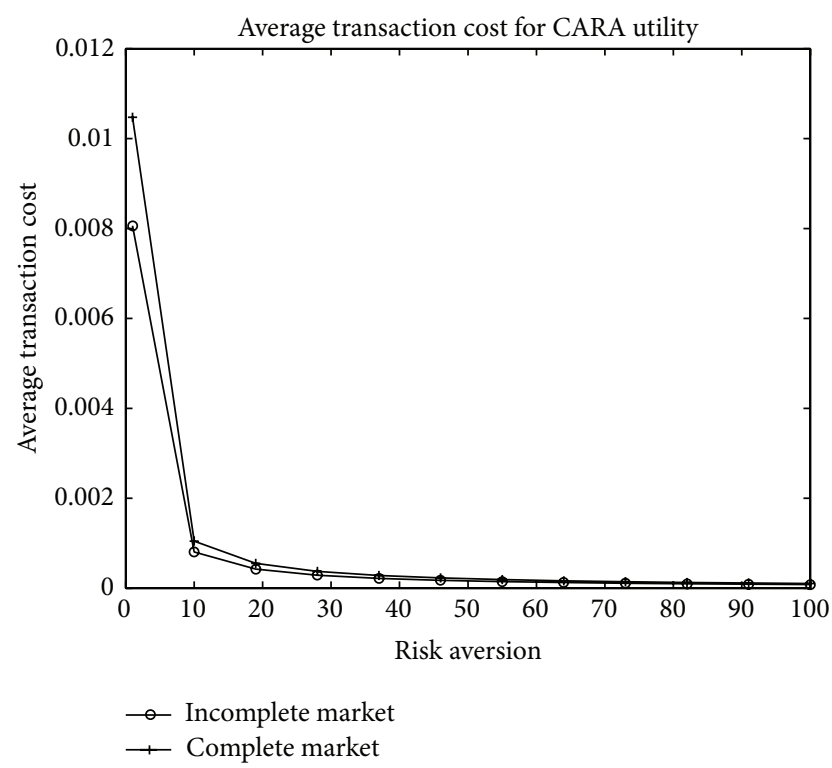

Figure 4: Average transaction cost for CARA utility in the two markets.

the ideal number of attainable stocks is 12 in the incomplete market. Thus an insurer will invest more in the only 10 stocks. An investor is venturesome in an incomplete market. Also we see that the total amount invested in stocks decreases as $\gamma$ increases. Since $\gamma$ is the absolute risk aversion coefficient, this result is consistent with intuition.

Figure 3 is a plot of the relative difference of the optimal strategy in an incomplete market with respect to that in a complete market; that is, it plots

$$
\frac{\left(\bar{\pi}_{\text {in }}-\bar{\pi}_{\mathrm{co}}\right)}{\bar{\pi}_{\mathrm{co}}}
$$

as a function of $\gamma$. The above $\bar{\pi}_{\text {in }}, \bar{\pi}_{\text {co }}$ denote the average total dollar amount invested in stocks in an incomplete market and in a complete market, respectively. It is obvious that this difference is independent of the risk aversion coefficient $\gamma$ but only related to the difference of the market structure. No matter what the risk aversion coefficients are, the effects of the incomplete market on the corresponding optimal strategies are the same.

Given a sequence of portfolios $\left\{\pi_{t}\right\}_{t=0}^{n}$, we quantify its average transaction cost by

$$
\frac{1}{n} \sum_{t=0}^{n-1}\left\|\pi_{t+1}-\pi_{t}\right\|_{1} .
$$

Figure 4 plots the average transaction costs of 12 periods optimal strategies for CARA utility in the two different markets. Same as the quadratic utility case, the cost of implementing the optimal investment in an incomplete market is lower than that in a complete market.

\section{Conclusion}

In this paper, we consider the optimal investment problem for an insurer in an incomplete market. After transforming the incomplete market into a complete one, we solve the problem via the martingale approach. From the computational experiments, we find that an insurer's optimal investment strategy in an incomplete market incurs lower transaction cost than the one in a complete market. The amount invested in stocks in an incomplete market is more than that in a complete market. For CARA utility, the computational results also show that the incomplete market produces the same effects on the optimal strategies of insurers with different risk aversion coefficients.

\section{Appendix}

See Tables 1 and 2.

\section{Acknowledgments}

The authors are very grateful to reviewers for their suggestions and this research was supported by the National Natural Science Foundation of China (Grant no. 11201335) and the Research Projects of the Social Science and Humanity on Young Fund of the Ministry of Education (Grant no. 11YJC910007). J. Cao would like to acknowledge the financial support from Auckland University of Technology during his sabbatical leave from July to December 2009 and thank the Department of Mathematics at Tianjin University for hospitality to host him in October 2009.

\section{References}

[1] S. Browne, "Optimal investment policies for a firm with a random risk process: exponential utility and minimizing the probability of ruin," Mathematics of Operations Research, vol. 20, no. 4, pp. 937-958, 1995.

[2] C. Hipp and M. Plum, "Optimal investment for insurers," Insurance, vol. 27, no. 2, pp. 215-228, 2000. 
[3] C. S. Liu and H. Yang, "Optimal investment for an insurer to minimize its probability of ruin," North American Actuarial Journal, vol. 8, no. 2, pp. 11-31, 2004.

[4] P. Azcue and N. Muler, "Optimal investment strategy to minimize the ruin probability of an insurance company under borrowing constraints," Insurance, vol. 44, no. 1, pp. 26-34, 2009.

[5] C. Hipp, "Stochastic control with application in insurance," in Stochastic Methods in Finance, vol. 1856 of Lecture Notes in Mathematics, pp. 127-164, Springer, Berlin, Germany, 2004.

[6] H. Yang and L. Zhang, "Optimal investment for insurer with jump-diffusion risk process," Insurance, vol. 37, no. 3, pp. 615634, 2005.

[7] Z. Wang, J. Xia, and L. Zhang, "Optimal investment for an insurer: the martingale approach," Insurance, vol. 40, no. 2, pp. 322-334, 2007.

[8] Q. Zhou, "Optimal investment for an insurer in the Lévy market: the martingale approach," Statistics \& Probability Letters, vol. 79, no. 14, pp. 1602-1607, 2009.

[9] I. Karatzas, J. P. Lehoczky, S. E. Shreve, and G.-L. Xu, "Martingale and duality methods for utility maximization in an incomplete market," SIAM Journal on Control and Optimization, vol. 29, no. 3, pp. 702-730, 1991.

[10] A. H. Zhang, "A secret to create a complete market from an incomplete market," Applied Mathematics and Computation, vol. 191, no. 1, pp. 253-262, 2007.

[11] N. Wang, "Optimal investment for an insurer with exponential utility preference," Insurance, vol. 40, no. 1, pp. 77-84, 2007.

[12] R. Cont and P. Tankov, Financial Modelling with Jump Processes, Chapman and Hall/CRC Financial Mathematics Series, CRC Press, Boca Raton, Fla, USA, 2003.

[13] S. E. Shreve, Stochastic Calculus for Finance. II: Continuous-time Models, Springer, Berlin, Germany, 2004.

[14] Z. Lu, "A computational study on robust portfolio selection based on a joint ellipsoidal uncertainty set," Mathematical Programming, vol. 126, no. 1, pp. 193-201, 2011. 


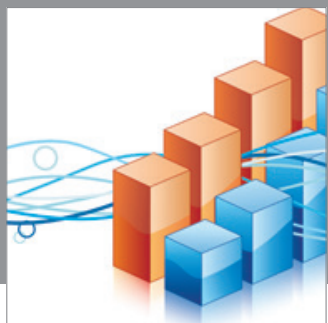

Advances in

Operations Research

mansans

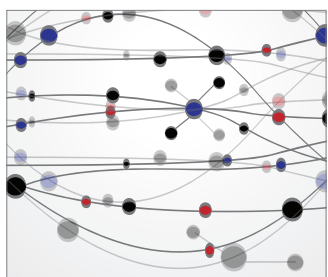

The Scientific World Journal
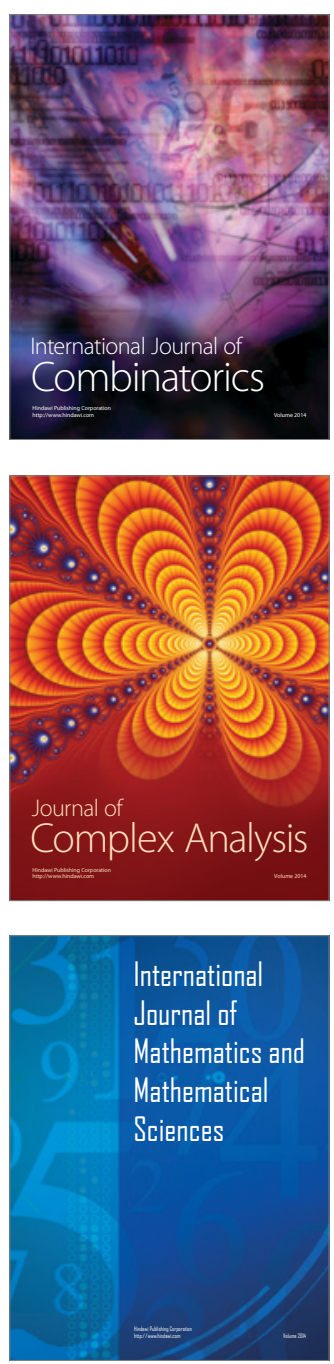
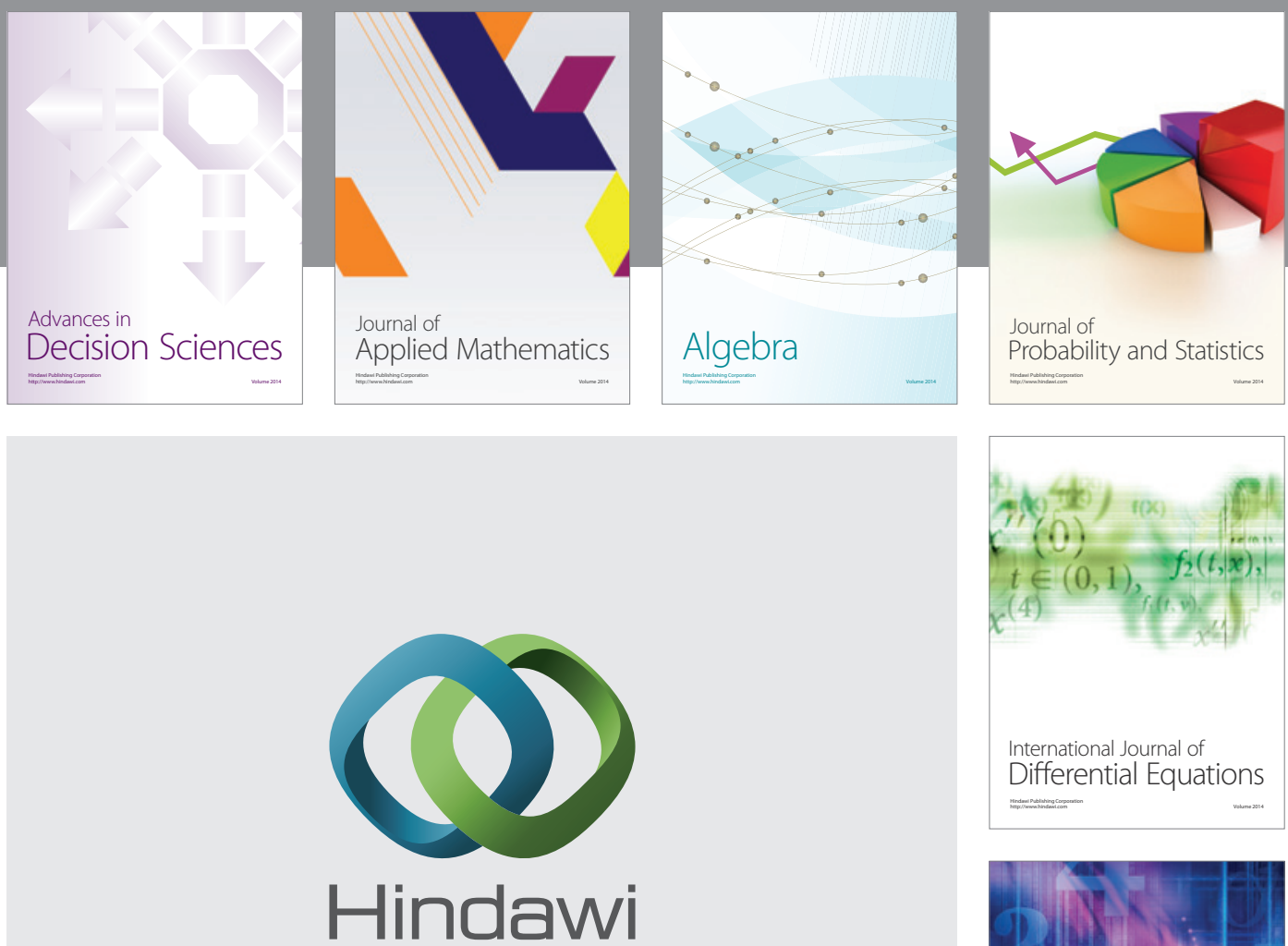

Submit your manuscripts at http://www.hindawi.com
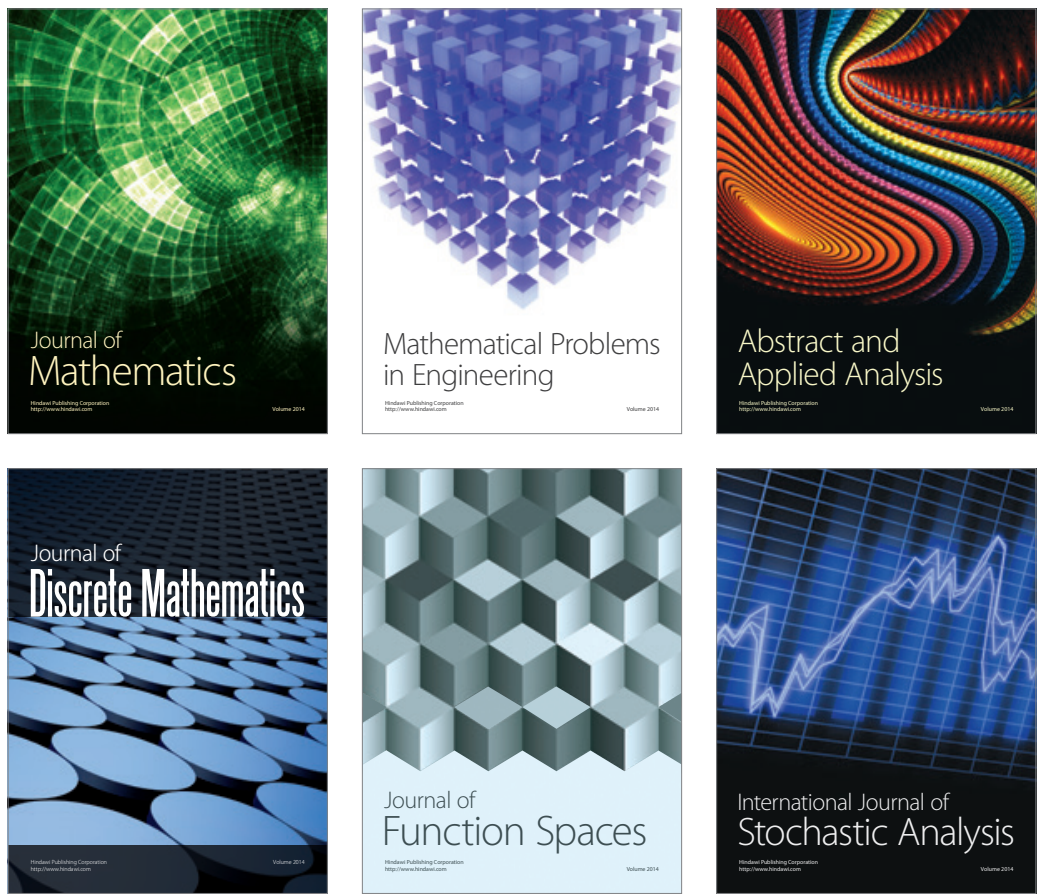

Journal of

Function Spaces

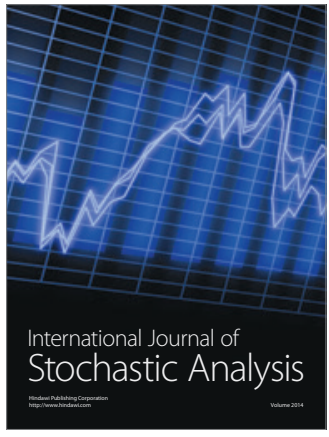

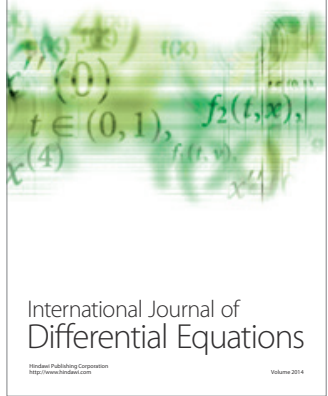
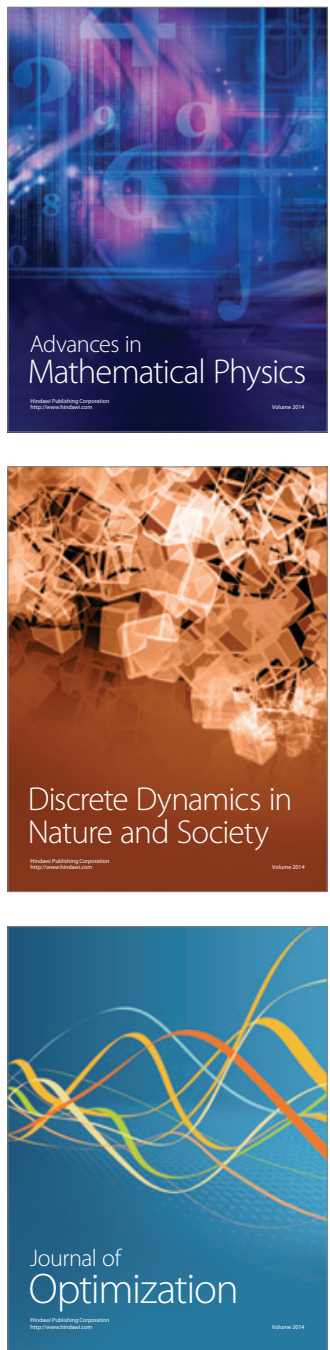